\title{
Avaliação Do Escore Atn-Iss Como Fator Prognóstico De Lesão Renal Aguda E Mortalidade Em Pacientes Internados Em Unidade De Terapia Intensiva;
}

\author{
Coronel, J.L.; Vidaletti, T.B.; Tonin, J.J.; Zambon, J.D.S.; Roza, L.E.M.; Amaral, \\ M.V.F.; Sobreira, L.S.; \\ Apresentador: Tamara Brun Vidaletti \\ Trabalho Premiado - $1^{\circ}$ Lugar \& Menção Honrosa - Destaque em Medicina Hospitalar
}

\section{Resumo}

Introdução: Lesão Renal Aguda (LRA) é tradicionalmente definida como a perda abrupta de função renal que resulta na retenção de ureia e outros resíduos nitrogenados, no desequilíbrio hidroeletrolítico e da taxa de filtração glomerular. Estimase que grande parte dos pacientes internados em Unidades de Terapia Intensiva (UTI) desenvolvem, pelo menos, formas leves de LRA, acarretando alta morbimortalidade e elevados custos em admissões em UTI. Diante disso, torna-se imprescindível o uso de escores prognósticos cada vez mais sensíveis e específicos. Alguns escores foram propostos, tal como o escore de APACHE II, que é amplamente aceito, porém sua especificidade é comprovadamente baixa em pacientes com LRA. Neste trabalho procuramos avaliar o escore ATN-ISS (acute tubular necrosis injury severity), publicado por Liaño em 1993, como fator prognóstico e de mortalidade nos pacientes com LRA internados na UTI do Hospital Universitário da Ulbra (HU). Método: Este é um estudo descritivo e retrospectivo, adquirido por revisão de prontuário, em que foram selecionados todos pacientes ingressos na UTI do HU com idades entre 22-89 anos no período de junho a agosto de $2012(\mathrm{n}=60)$. Os pacientes foram divididos em 2 grupos, aqueles sem LRA e os com LRA ( $\mathrm{n}=16)$, sendo considerados com LRA aqueles com creatinina sérica maior que $1,5 \mathrm{mg} / \mathrm{dL}$ ou elevação de mais de $50 \%$ acima do valor basal em um período de $24 \mathrm{hs}$. Como variáveis demográficas foram avaliadas idade e gênero, além de motivo e tempo de internação. Informações específicas, tais como causa de LRA, e tempo de permanência na UTI, também foram avaliados. Resultados: Em relação ao escore ATN-ISS, houve diferença estatisticamente significativa para discriminação entre pacientes que desenvolveram LRA e os que não desenvolveram. Observou-se que a partir de 0,638 no escore de ATN-ISS todos os pacientes apresentaram evolução para LRA. Esta população, durante o período de observação, apresentou uma chance de mortalidade cinco vezes maior do que a população controle com um $\mathrm{RR}=$ 5,04 (IC 95\% 2.48 - 10.4), com um $\mathrm{P}<0.0001$. Este escore apresentou elevadas sensibilidade e especificidade para discriminação de desfecho de LRA e mortalidade $(85,7 \%$ e $97,5 \%$, e $65 \%$ e $97,5 \%$ respectivamente). Conclusão: O escore ATN-ISS, é um preditor de risco de LRA e mortalidade, sendo um método discriminatório de desfecho simples e útil na prática clínica, apresentando elevada sensibilidade e especificidade em termos de prognóstico.

\section{Referência:}

Coronel, J.L.; Vidaletti, T.B.; Tonin, J.J.; Zambon, J.D.S.; Roza, L.E.M.; Amaral, M.V.F.; Sobreira, L.S.; Avaliação Do Escore Atn-Iss Como Fator Prognóstico De Lesão Renal Aguda E Mortalidade Em Pacientes Internados Em Unidade De Terapia Intensiva; . In: II Congresso Brasileiro de Medicina Hospitalar - II CBMH [= Blucher Medical Proceedings, vol.1, num.5] São Paulo: Editora Blucher, 2014. p.1

DOI 10.5151/medpro-II-cbmh-076 